\title{
Effect of carbamazepine on dolutegravir pharmacokinetics and dosing recommendation
}

\author{
Ivy Song $^{1} \cdot$ Steve Weller $^{1} \cdot$ Juhin Patel $^{1} \cdot$ Julie Borland $^{1} \cdot$ Brian Wynne $^{1}$. \\ Mike Choukour $^{1} \cdot$ Fred Jerva $^{1} \cdot$ Stephen Piscitelli $^{1}$
}

Received: 6 November 2015 / Accepted: 1 February 2016/Published online: 22 February 2016

(C) The Author(s) 2016. This article is published with open access at Springerlink.com

\begin{abstract}
Purpose Dolutegravir (DTG) is primarily metabolized by UGT1A1 with CYP3A as a minor route. Carbamazepine (CBZ) is a potent inducer of these enzymes; thus, the effect of oral extended-release CBZ on DTG pharmacokinetics (PK) was evaluated to provide dose recommendation when coadministered.

Methods This was a single-center, open-label, fixed-sequence, crossover study in healthy adults. Subjects received three treatments: DTG $50 \mathrm{mg}$ every $24 \mathrm{~h}(\mathrm{q} 24 \mathrm{~h}) \times 5$ days in period 1, followed by CBZ $100 \mathrm{mg}$ every $12 \mathrm{~h}$ $(\mathrm{q} 12 \mathrm{~h}) \times 3$ days, then $200 \mathrm{mg} \mathrm{q} 12 \mathrm{~h} \times 3$ days, then $300 \mathrm{mg}$ $\mathrm{q} 12 \mathrm{~h} \times 10$ days in period 2, and DTG $50 \mathrm{mg}$ q24h $+\mathrm{CBZ}$ $300 \mathrm{mg} \mathrm{q} 12 \mathrm{~h} \times 5$ days in period 3 . No washout intervals occurred. Each dose was administered with a moderate-fat meal. Serial PK samples for DTG were collected on day 5 of periods 1 and 3. Plasma DTG PK parameters were determined with non-compartmental analysis. Geometric least-squares mean ratios (GMRs) and $90 \%$ confidence intervals (CIs) were generated by the mixed-effect model for within-subject treatment comparisons. Safety assessments were performed throughout the study.

Results Sixteen subjects enrolled; 14 completed the study. CBZ significantly reduced DTG exposure: GMRs $(90 \% \mathrm{CI})$ for DTG + CBZ versus DTG alone were $0.51(0.48-0.549)$, $0.67(0.61-0.73)$, and $0.27(0.24-0.31)$ for area under the curve from time zero to the end of the dosing interval $(\operatorname{AUC}(0-\tau))$, maximum observed plasma concentration
\end{abstract}

Brian Wynne

brian.r.wynne@viivhealthcare.com

1 GlaxoSmithKline, Research Triangle Park, Durham, NC, USA
(Cmax), and plasma concentration at the end of the dosing interval $(\mathrm{C} \tau)$, respectively. DTG alone and co-administered with CBZ was well tolerated.

Conclusion Integrase strand transfer inhibitor-naive subjects taking CBZ should receive DTG $50 \mathrm{mg}$ twice daily versus once daily, as is recommended with other potent UGT1A/ CYP3A inducers.

ClinicalTrials.gov: NCT01967771

Keywords Carbamazepine - Dolutegravir · Drug interaction · Healthy subjects

\section{Introduction}

Dolutegravir (DTG, Tivicay ${ }^{\circledR}$, ViiV Healthcare) is an HIV integrase strand transfer inhibitor approved for use in combination with other antiretrovirals for the treatment of HIV infection in adults and adolescents. The usual dose in integrase strand transfer inhibitor-naive subjects is $50 \mathrm{mg}$ once daily [1]. Dolutegravir is a substrate of P-glycoprotein (P-gp) and is primarily metabolized by uridine diphosphate glucuronosyltransferase (UGT) 1A1 with a minor component (approximately $10 \%$ ) via cytochrome P450 (CYP) 3A4 [1, 2]. Clinically significant drug interactions requiring a dose adjustment with DTG have not been observed with inhibitors of UGT1A1, CYP3A4, or P-gp [3, 4]. However, a clinically significant decrease in exposure has been observed with DTG when co-administered with the strong CYP3A4 inducers that include tipranavir/ritonavir, efavirenz, and rifampin $[5,6]$. These enzyme inducers require the dose of DTG to be increased from $50 \mathrm{mg}$ once daily to $50 \mathrm{mg}$ twice daily.

Neurologic manifestations of HIV infection are quite diverse, and many individuals with HIV infection who are taking antiretroviral therapy require treatment with 
anticonvulsants. Anticonvulsant medications are commonly used in patients with HIV either for seizure management or for expanded indications such as neuropathic pain and psychiatric disorders, including depression [7, 8]. Carbamazepine (CBZ) is an antiepileptic drug (AED) that has been used for the treatment of depression as well as trigeminal neuralgia. Carbamazepine is a potent enzyme inducer and is known to induce its own metabolism, resulting in a shorter half-life (12-17 h) and lower exposure following chronic dosing compared with the half-life $(25-65 \mathrm{~h})$ and exposure following single dosing [11]. In addition, potent induction of CYP3A4 and UGT1A1 enzymes by CBZ can lead to underexposure of drugs that are metabolized by these enzymes $[9,10]$. Considering the metabolic pathways of DTG and CBZ and that CBZ may be prescribed to HIV-infected subjects, an evaluation of the effects of CBZ on DTG pharmacokinetics (PK) was warranted.

In vitro, DTG demonstrated minimal or no direct inhibition of CYP isozymes, UGT1A1, UGT2B7, and many transporters (P-gp, breast cancer resistance protein, OATP1B1, OATP1B3, MRP2) and was not an inducer of CYP1A2, CYP2B6, or CYP3A4 [1, 2]. Dolutegravir also had no significant effect on midazolam exposure, a probe substrate for CYP3A4, in healthy subjects [1]. Carbamazepine is primarily metabolized in the liver by CYP3A. However, based on DTG enzyme interaction profile, it was not expected to affect CBZ PK; thus, the primary objective of this study was to evaluate the effect of CBZ on DTG PK in healthy subjects and not vice versa.

\section{Methods}

\section{Study design}

This was a phase I, single-site, open-label, fixed-sequence, crossover study with three treatment periods to evaluate the effect of an oral extended-release formulation of CBZ on the steady-state PK of orally administered DTG in 16 healthy subjects. The study was conducted in accordance with the principles of the Declaration of Helsinki. A written informed consent was obtained from all subjects, and the protocol was approved by the institutional review board of the study site. The trial was registered at ClinicalTrials.gov (NCT01967771; protocol number: 200901).

Each subject was evaluated with physical examination, medical history, and laboratory testing at a screening visit conducted within 30 days prior to the first dose of drug and at a follow-up visit 7 to 14 days after the last dose of study drug. Eligible subjects were 18 to 65 years of age, had a minimum weight requirement of $45 \mathrm{~kg}$ for women and $50 \mathrm{~kg}$ for men, and a body mass index requirement of between 18.5 and $31.0 \mathrm{~kg} / \mathrm{m}^{2}$. Subjects with positive pre-study drug/alcohol screens, positive HIV antibody test results, and positive serum or urine human chorionic gonadotropin test results for pregnancy, as well as Asians with positive HLAB*1502 allele (carriage is associated with a risk of hypersensitivity reactions with exposure to CBZ) and subjects with allergies to tricyclic antidepressants, were excluded. Women of child-bearing potential agreed to protocol-specified methods of contraception to avoid pregnancy. Subjects were not allowed to receive prescription or non-prescription/herbal medications within 7 days of dosing or throughout the study. Use of antacids, vitamins, or calcium or iron supplements, which are known to reduce DTG absorption, was strictly prohibited within $24 \mathrm{~h}$ before the first dose of study medication and throughout the trial, including follow-up. Acetaminophen was permitted with a dose that did not exceed $2 \mathrm{~g}$ per day.

During period 1 , subjects received DTG $50 \mathrm{mg}$ once daily as a single agent for days 1 to 5 . During period 2, subjects received CBZ alone and the dose was escalated: $100 \mathrm{mg}$ twice daily for days 1 to 3, $200 \mathrm{mg}$ twice daily for days 4 to 6 , and $300 \mathrm{mg}$ twice daily for days 7 to 16 . During period 3, DTG $50 \mathrm{mg}$ once daily was co-administered with CBZ $300 \mathrm{mg}$ twice daily for days 1 to 5 . There was no washout between treatment periods. As CBZ is recommended to be given with food per product information [11], all doses in the testing unit, including those on PK collection days, were administered orally in the morning $30 \mathrm{~min}$ after the start of the moderatefat meal, and subjects were asked to do the same when dosing outside the unit. Subjects were housed at the clinical research unit from period 1 , day 1 , until the morning of period 2 , day 8 , as well as from period 2, day 16 , through period 3, day 6 . Phone call reminders and pill counts were employed when subjects self-administered CBZ as outpatients during period 2 , days 8 to 16 . Serial blood samples for determining the plasma concentration of DTG were collected on day 5 of period 1 and day 16 of period 3 at the following times: predose (within 15 min before study dose), 0.5, 1, 2, 3, 4, 6, 12, and $24 \mathrm{~h}$ after DTG dosing.

The DTG dose selected for this study was the $50 \mathrm{mg}$ oncedaily dose regimen recommended for the treatment of HIV integrase inhibitor (INI)-naive subjects. A higher dose of $50 \mathrm{mg}$ twice daily is recommended for subjects with INI resistance; however, this population represents a small portion of HIV-infected subjects to be treated with DTG. Therefore, the more common once-daily dose was evaluated in this study. Carbamazepine dose selection was based on the range of likely CBZ clinical doses [11], the time course and magnitude of CBZ induction effect observed in other studies [10, 12], and consideration of the risks/benefits of CBZ administration to healthy subjects [11]. Review of prior CBZ drug-interaction studies found most CYP3A/UGT1A1 substrate drugs demonstrated substantially altered PK when CBZ was dosed between 200 and $600 \mathrm{mg}$ daily for 3 weeks or less. Carbamazepine is titrated to achieve seizure or pain control, with clinically 
effective (adult) doses ranging from $400 \mathrm{mg}$ up to a maximum of $1000 \mathrm{mg}$ daily. There is no benefit, and only theoretical risk, to dosing volunteers with a central nervous systemactive drug such as CBZ. For this reason, in this study, CBZ was titrated upward over a 7-day period to a mid-range value of $600 \mathrm{mg}$ daily, for a total of 21 days of CBZ exposure, to allow for complete or near complete induction. The druginteraction PK assessment was obtained on the last day of period 3 (day 21).

\section{Safety evaluations}

Safety evaluations included the monitoring of adverse events (AEs), physical examinations, clinical chemistry laboratory tests, vital signs, and electrocardiograms. Additionally, assessment of Columbia suicide severity rating scale scores was performed throughout the study, because CBZ exposure (along with exposure to several other AEDs) has been associated with increased risk of suicidal ideation and depression.

\section{Bioanalytical methods}

Plasma samples were analyzed for DTG by PPD, Inc. (Middleton, WI) using a validated analytical method based on protein precipitation with acetonitrile containing the stable isotope-labeled internal standard (GSK1349572- $\mathrm{d}_{7-}{ }^{15} \mathrm{~N}$ ), followed by high-performance liquid chromatography/ tandem mass spectrometry analysis. The lower limit of quantification for DTG in plasma was $20 \mathrm{ng} / \mathrm{mL}$, and the upper limit of quantification was $20,000 \mathrm{ng} / \mathrm{mL}$. Precision and accuracy were evaluated by replicate analyses of human plasma quality control samples prepared at five concentrations: 60, 160, 640, 2400, and 15,200 ng/mL. Precision, measured as the percent coefficient of variation, ranged from 3.0 to $5.9 \%$ across the quality control range. Accuracy, expressed as the percent difference from the mean value, ranged from -2.2 to $1.8 \%$. Both were within acceptance standards of $15 \%$.

\section{Pharmacokinetic analysis}

A non-compartmental PK analysis of the DTG concentrationtime data was conducted by Phast Clinical Data, Inc. (Raleigh, NC). Pharmacokinetic analyses of plasma DTG concentration-time data were analyzed using Model 200 (for extravascular administration) of Phoenix WinNonlin version 6.3 (Pharsight Corporation, St. Louis, MO). Plasma PK parameters for DTG were calculated using actual elapsed times from dosing. The individual PK parameters that were determined included area under the curve from time zero to the end of the dosing interval (AUC $(0-\tau)$ ), maximum observed plasma concentration $(\mathrm{Cmax})$, plasma concentration at the end of the dosing interval $(\mathrm{C} \tau)$, oral clearance, and terminal half-life.

\section{Statistical analysis}

This study was designed to estimate the magnitude of druginteraction effect of $\mathrm{CBZ}$ on the PK parameters of DTG. No formal hypothesis was tested. Instead, an estimation approach was used to evaluate the effect of CBZ on DTG PK. Pharmacokinetic parameters were log-transformed and analyzed by analysis of variance to determine the point estimate and associated $90 \%$ confidence intervals for the difference between test treatment (DTG + CBZ) and reference treatment (DTG alone). These values were then back-transformed to calculate the point and interval estimates for test-to-reference treatment ratios on the original scale. Geometric least-squares mean ratios and $90 \%$ confidence intervals were generated by the mixed-effect model for within-subject treatment comparisons.

\section{Results}

\section{Demographics}

Sixteen subjects, 2 females and 14 males, were enrolled and included in the safety and PK populations. Two subjects were prematurely discontinued from the study: one male subject withdrew due to pyrexia/febrile illness on period 2, day 3 (CBZ alone), and one female subject withdrew due to druginduced hypersensitivity syndrome, increased alanine aminotransferase (ALT) level, thrombocytopenia, and maculopapular rash in period 2, day 11 (CBZ alone). The mean (standard deviation (SD)) age was 38.6 (12.8) years and ranged between 18 and 65 years. The mean (SD) body mass index was $26.1(3.2) \mathrm{kg} / \mathrm{m}^{2}$, height was $177.8(6.3) \mathrm{cm}$, and weight was $82.4(11.5) \mathrm{kg}$. Fifteen subjects (94\%) were not Hispanic or Latino. Six subjects (38\%) were African American or of African heritage, while 10 subjects (63\%) were White/Caucasian or of European heritage.

\section{Safety}

No subjects experienced AEs during period 1 (DTG alone). Seven subjects experienced at least one AE: six subjects while taking CBZ alone and one subject in the DTG + CBZ group (Table 1). One subject in the DTG + CBZ group experienced a photosensitivity event to overhead fluorescent light that occurred during period 3 and resolved without interruption of drug administration within 5 days. Headache, thrombocytopenia, and nausea were the only AEs that occurred in more than one subject. No grade 3 or grade 4 AEs, deaths, or non-fatal serious AEs were reported. Two subjects were permanently discontinued because of AEs in period 2 ( $\mathrm{CBZ}$ alone). One male subject developed a febrile illness after receiving $\mathrm{CBZ}$ in period 2 . His lymphocyte count dropped to $0.5 \times 10^{-3}$ cells/ 
Table 1 Summary of drugrelated adverse events by treatment

\begin{tabular}{llll}
\hline & $\begin{array}{l}\text { DTG alone }(n=16) \\
n(\%)\end{array}$ & $\begin{array}{l}\text { CBZ alone }(n=16) \\
n(\%)\end{array}$ & $\begin{array}{l}\text { DTG + CBZ }(n=14) \\
n(\%)\end{array}$ \\
\hline Any event & 0 & $6(38)$ & $1(7)$ \\
Asthenia & 0 & $1(6)$ & 0 \\
Fatigue & 0 & 0 & $1(7)$ \\
Pyrexia & 0 & $1(6)$ & 0 \\
Thirst & 0 & $1(6)$ & 0 \\
Headache & 0 & $2(13)$ & 0 \\
Cognitive disorder & 0 & $1(6)$ & 0 \\
Thrombocytopenia & 0 & $2(13)$ & 0 \\
Leukopenia & 0 & $1(6)$ & 0 \\
Lymphopenia & 0 & $1(6)$ & 0 \\
Drug reaction with eosinophilia & 0 & $1(6)$ & 0 \\
and systemic symptoms & & 0 & $1(7)$ \\
Photosensitivity reaction & 0 & $1(6)$ & 0 \\
Rash maculopapular & 0 & $1(6)$ & 0 \\
Nausea & 0 & $1(6)$ & 0 \\
ALT increased & 0 & $1(6)$ & 0 \\
Hyponatremia & 0 & $1(6)$ & 0 \\
Pain in extremity & 0 & $1-5):(0 B Z$ & \\
\hline
\end{tabular}

Treatments: DTG alone = DTG $50 \mathrm{mg}$ once daily $($ period 1 , days $1-5) ; \mathrm{CBZ}=\mathrm{CBZ} 100 \mathrm{mg}$ twice daily $($ period 2, days 1-3) + CBZ $200 \mathrm{mg}$ twice daily (period 2, days 4-6) + CBZ $300 \mathrm{mg}$ twice daily (period 2, days 7-16); $\mathrm{DTG}+\mathrm{CBZ}=\mathrm{DTG} 50 \mathrm{mg}$ once daily $+\mathrm{CBZ} 300 \mathrm{mg}$ twice daily (period 3, days $1-5)$

$A L T$ alanine aminotransferase, $C B Z$ carbamazepine, $D T G$ dolutegravir $\mathrm{mm}^{3}$, and ALT increased to $66 \mathrm{IU} / \mathrm{L}$ (grade 1), which was thought to be an unrelated viral infection. The second subject was a 23-year-old Caucasian female who developed a suspected moderate (grade 2) hypersensitivity syndrome that manifested with a facial rash and headache while she was receiving $300 \mathrm{mg} \mathrm{CBZ}$ twice daily during period 2 . Laboratory analysis revealed a decrease in white blood cell count to 3800 cells $/ \mathrm{mm}^{3}$ (compared with 8800 cells $/ \mathrm{mm}^{3}$ at baseline), a decrease in platelet count to a low of 78,000 cells/ $\mathrm{mm}^{3}$ (from 159,000 3 days before), and an increase in ALT level to a high of $151 \mathrm{IU} / \mathrm{L}$ (grade 2), while bilirubin levels remained within normal limits. This was considered possibly related to CBZ. The rash slowly resolved over the next 2 weeks, and all laboratory abnormalities in this subject resolved within 3 to 4 weeks of CBZ discontinuation.

\section{Pharmacokinetics}

Pharmacokinetic parameters following repeat-dose administration of DTG with and without CBZ and treatment comparisons are shown in Table 2, and the mean (SD) concentrationtime profiles are shown in Fig. 1. The geometric mean
Table 2 Summary and statistical comparison of plasma DTG PK parameters following DTG $50 \mathrm{mg}$ once-daily dose administration with and without CBZ

\begin{tabular}{llll}
\hline PK parameter & \multicolumn{2}{l}{ Geometric mean $(\mathrm{CV} \%)$} & \multirow{2}{*}{ Ratio of GLS means $(90 \% \mathrm{CI})$} \\
\cline { 2 - 3 } & DTG alone $(n=16)$ & $\mathrm{DTG}+\mathrm{CBZ}(n=14)$ & DTG + CBZ vs DTG alone \\
\hline $\mathrm{AUC}(0-\tau)(\mu \mathrm{g} \cdot \mathrm{h} / \mathrm{mL})$ & $53.8(21.4)$ & $27.4(22.1)$ & $0.512(0.477,0.549)$ \\
$\mathrm{Cmax}(\mu \mathrm{g} / \mathrm{mL})$ & $4.16(14.4)$ & $2.77(25.0)$ & $0.666(0.610,0.726)$ \\
$\mathrm{C} \tau(\mu \mathrm{g} / \mathrm{mL})$ & $1.20(39.1)$ & $0.325(45.9)$ & $0.274(0.240,0.313)$ \\
$\mathrm{CL} / \mathrm{F}(\mathrm{L} / \mathrm{h})$ & $0.929(21.4)$ & $1.83(22.1)$ & $1.95(1.82,2.10)$ \\
$\mathrm{t} 1 / 2(\mathrm{~h})$ & $12.9(23.8)$ & $7.31(16.9)$ & $0.567(0.526,0.611)$ \\
\hline
\end{tabular}

Treatments: DTG alone $=$ DTG 50 mg once daily; DTG + CBZ $=$ DTG 50 mg once daily + CBZ 300 mg twice daily

$A U C(0-\tau)$ area under the curve from time zero to the end of the dosing interval, $C \tau$ concentration at the end of the dosing interval, $C B Z$ carbamazepine, $C I$ confidence interval, $C L / F$ oral clearance, $C$ max maximum observed plasma concentration, $C V \%$ coefficient of variation, $D T G$ dolutegravir, $G L S$ geometric least squares, $P K$ pharmacokinetic, $t 1 / 2$ terminal elimination half-life 
Fig. 1 Mean \pm SD concentrationtime profile for dolutegravir $(D T G)$ with and without concomitant administration of carbamazepine (CBZ). Treatment: DTG alone $=$ DTG $50 \mathrm{mg}$ once daily; DTG + CBZ = DTG $50 \mathrm{mg}$ once daily + CBZ $300 \mathrm{mg}$ twice daily

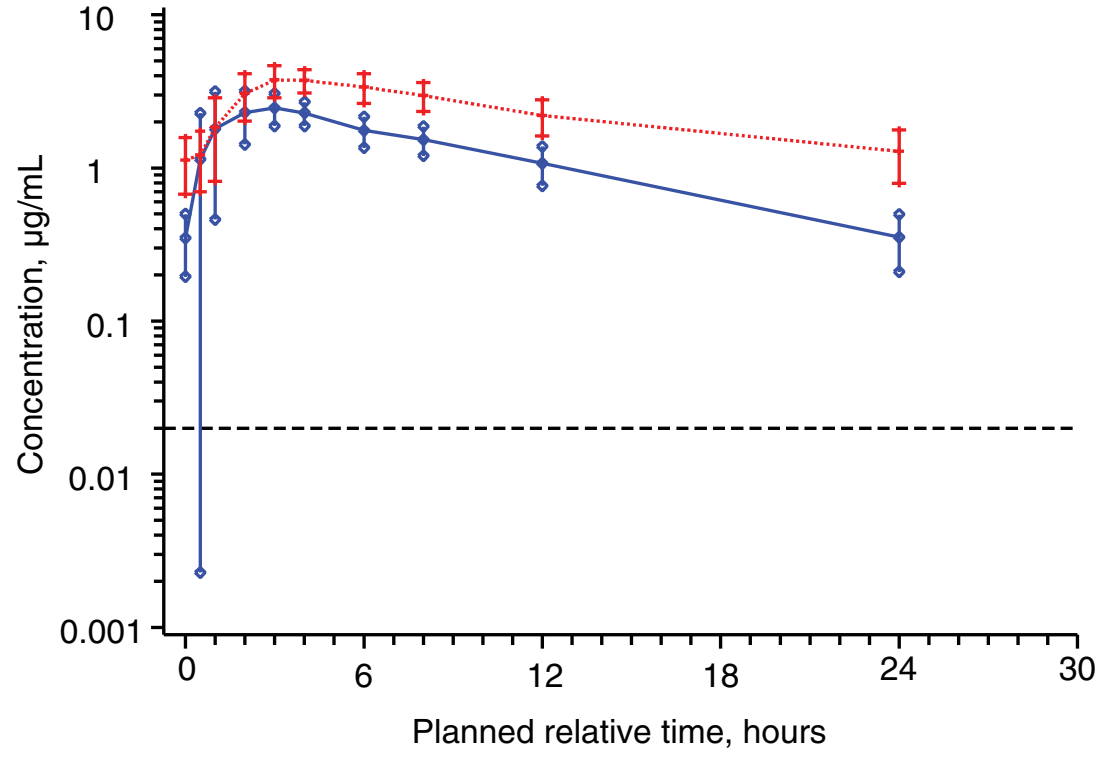

DTG alone $+-+-+\quad \mathrm{DTG}+\mathrm{CBZ} \diamond \diamond \diamond$
AUC(0- $\tau)$, Cmax, and C $\tau$ of DTG were reduced by 49, 33, and $73 \%$, respectively, when DTG was co-administered with CBZ. Dolutegravir clearance was increased by $95 \%$, and its half-life shortened from 12.9 to $7.31 \mathrm{~h}$ when it was coadministered with $\mathrm{CBZ}$. On an individual subject basis, the reduction in $\mathrm{AUC}(0-\tau)$ ranged from 27 to $58 \%$, and the reduction in $\mathrm{C} \tau$ ranged from 53 to $82 \%$ for the 14 subjects with PK results during both treatment periods.

\section{Discussion}

Carbamazepine is a potent inducer of CYP3A4 and UGT1A1 enzymes and has been shown to decrease plasma concentrations of a number of drugs [9-13]. This study demonstrated that repeated dosing of CBZ for 21 days resulted in significant reductions of 49, 33, and $73 \%$ in $\mathrm{AUC}(0-\tau), \mathrm{Cmax}$, and $\mathrm{C} \tau$, respectively, of DTG. Dolutegravir is primarily metabolized to an inactive ether glucuronide by UGT1A1, with some minor oxidative metabolism by CYP3A4; therefore, the increased DTG clearance observed in this study is likely a result of concurrent UGT1A1 and CYP3A4 induction by CBZ. Although regulatory guidance generally recommends that the highest possible clinical dose be used in the evaluation of a drug as a perpetrator of drug interactions, a lower but more commonly administered mid-range CBZ dose regimen (titration up to $600 \mathrm{mg} /$ day) was selected for this study to minimize adverse effects in the healthy subject population. Based on the results of prior studies at the $600 \mathrm{mg} /$ day or lower doses $[10,12]$, the magnitude of induction observed in this study was likely near maximal or maximal and provided ample data for dose adjustment decisions.

The degree of induction observed in this study of CBZ and DTG was similar to that reported when DTG was coadministered with other potent CYP450 enzyme inducers such as tipranavir/ritonavir, efavirenz, and rifampin, which reduced DTG AUC(0- $\tau)$ in the range of 36 to $49 \%$ and $\mathrm{C} \tau$ by 72 to $76 \%[5,6]$. The substantial reduction in $\mathrm{C} \tau$ observed in these prior studies was considered clinically significant based on the DTG exposure-antiviral response relationship and observation that subjects receiving inducers in a phase III trial had lower antiviral response rates [14]. Therefore, the initial product labeling recommended an upward dose adjustment from $50 \mathrm{mg}$ once daily to $50 \mathrm{mg}$ twice daily when DTG is being co-administered with tipranavir/ritonavir, efavirenz, and rifampin in INI-naive subjects [1]. The present study showed that another strong enzyme inducer, CBZ, decreased DTG $\mathrm{C} \tau$ to a similar degree $(\sim 73 \%)$ as tipranavir/ ritonavir, efavirenz, and rifampin. Therefore, the same dose adjustment recommendation for DTG should be applied to CBZ. As with efavirenz and tipranavir/ritonavir, it is expected that DTG $50 \mathrm{mg}$ twice daily co-administered with CBZ will demonstrate similar, if not higher, virologic response as that observed in subjects receiving DTG $50 \mathrm{mg}$ once daily without these inducers. In INI-resistant subjects, where the exposures of the higher $50 \mathrm{mg}$ twice-daily regimen are required for antiviral activity against resistant virus, co-administration with these strong enzyme inducers should be avoided [1].

Antiepileptic drugs are a diverse group of pharmacological agents and can generally be classified into two groups based on enzyme induction potential: enzyme-inducing and noninducing AEDs [12]. Although unstudied, the enzymeinducing AEDs such as phenytoin, oxcarbazepine, and 
phenobarbital are also expected to decrease DTG exposures and require DTG dose adjustment to $50 \mathrm{mg}$ twice daily. Use of non-inducing AEDs such as gabapentin, lamotrigine, levetiracetam, tiagabine, and topiramate may be considered as an alternative to inducing drugs like CBZ to avoid DTG dose adjustment.

\section{Conclusion}

This study evaluated the magnitude of a drug-drug interaction between CBZ, a known inducer of UGT1A1 and CYP3A, and DTG, a known substrate of UGT1A1 and CYP3A. As expected, co-administration of DTG and CBZ in healthy subjects resulted in a potentially clinically significant decrease in DTG AUC $(0-\tau)$, Cmax, and C $\tau$ compared with DTG administered alone. Based on accumulated PK-pharmacodynamic relationship data regarding DTG and the currently approved dosing recommendation with other strong inducers, DTG dose adjustment to $50 \mathrm{mg}$ twice daily is recommended when DTG is co-administered with CBZ.

Acknowledgments All listed authors meet the criteria for authorship set forth by the International Committee for Medical Journal Editors. The authors wish to acknowledge the following individuals for editorial assistance during the development of this manuscript: Kim Adkison, John Fuchs, and Meredith MacPherson.

Contributions of authors Ivy Song, Steve Weller, Juhin Patel, Julie Borland, Brian Wynne, Fred Jerva, and Mike Choukour were involved in the study design; the preparation and/or review of the protocol, data analysis plan, results, and study report; and the preparation of this manuscript. Steve Piscitelli was involved in the review of the study design, protocol, and study report and in the preparation of the manuscript.

\section{Compliance with ethical standards}

Funding This study was funded by ViiV Healthcare.

Conflicts of interest All authors were employees of and had stock/ stock options in GlaxoSmithKline at the time the work described in the manuscript was done.

Ethical approval All procedures performed in studies involving human participants were in accordance with the ethical standards of the institutional and/or national research committee and with the 1964 Helsinki declaration and its later amendments or comparable ethical standards. A written informed consent was obtained from all subjects, and the protocol was approved by the institutional review board of the study site.

Open Access This article is distributed under the terms of the Creative Commons Attribution 4.0 International License (http:// creativecommons.org/licenses/by/4.0/), which permits unrestricted use, distribution, and reproduction in any medium, provided you give appropriate credit to the original author(s) and the source, provide a link to the Creative Commons license, and indicate if changes were made.

\section{References}

1. Tivicay $^{\circledR}$ (dolutegravir) [package insert]. Research Triangle Park: ViiV Healthcare; 2014

2. Reese MJ, Savina PM, Generaux GT, Tracey H, Humphreys JE, Kanaoka E, Webster LO, Harmon KA, Clarke JD, Polli JW (2013) In vitro investigations into the roles of drug transporters and metabolizing enzymes in the disposition and drug interactions of dolutegravir, a HIV integrase inhibitor. Drug Metab Dispos 41: 353-361

3. Song I, Min SS, Borland J, Lou Y, Chen S, Patel P, Ishibashi T, Piscitelli SC (2011) The effect of lopinavir/ritonavir and darunavir/ ritonavir on the HIV integrase inhibitor S/GSK1349572 in healthy participants. J Clin Pharmacol 51:237-242

4. Song I, Borland J, Chen S, Lou Y, Peppercorn A, Wajima T, Min S, Piscitelli SC (2011) Effect of atazanavir and atazanavir/ritonavir on the pharmacokinetics of the next-generation HIV integrase inhibitor, S/GSK1349572. Br J Clin Pharmacol 72:103-108

5. Song I, Borland J, Chen S, et al. (2014) Effects of enzyme inducers efavirenz and tipranavir/ritonavir on the pharmacokinetic of the HIV integrase inhibitor dolutegravir. Eur J Clin Pharmacol 70: 1173-1179

6. Dooley K, Sayre P, Borland J, et al. (2013) Safety, tolerability, and pharmacokinetics of the HIV integrase inhibitor dolutegravir given twice daily with rifampin or once daily with rifabutin: results of a phase 1 study among healthy subjects. J Acquir Immune Defic Syndr 62:21-27

7. Kellinghaus C, Engbring C, Kovac S, et al. (2008) Frequency of seizures and epilepsy in neurological HIV-infected patients. Seizure 17:27-33

8. McArthur JC, Brew BJ, Nath A (2005) Neurological complications of HIV infection. Lancet Neurol 4:543-555

9. Oscarson M, Zanger UM, Rifki OF, Klein K, Eichelbaum M, Meyer UA (2006) Transcriptional profiling of genes induced in the livers of patients treated with carbamazepine. Clin Pharmacol Ther 80:440-456

10. Spina E, Pisani F, Perucca E (1996) Clinically significant pharmacokinetic drug interactions with carbamazepine: an update. Clin Pharmacokinet 31:198-214

11. Tegretol $^{\circledR}$ (carbamazepine) [package insert]. East Hanover: Novartis; 2014

12. Anderson GD (2004) Pharmacogenetics and enzyme induction/ inhibition properties of antiepileptic drugs. Neurology 63:S3-S8

13. Brodie MJ, Mintzer S, Pack AM, Gidal BE, Vecht CJ, Schmidt D (2013) Enzyme induction with antiepileptic drugs: cause for concern? Epilepsia 54:11-27

14. Song I, Chen S, Piscitelli S, Min S. Pharmacokinetics (PK) and PKpharmacodynamic (PD) relationship of dolutegravir (DTG) in INInaive subjects. Abstract A-1573. Presented at: 53rd Interscience Conference on Antimicrobial Agents and Chemotherapy; 2013; Denver 